\title{
Cost-Effectiveness Analysis for the Treatment of Diabetic Foot Ulcer in France: Platelet-Rich Plasma vs Standard of Care
}

\author{
Salvatore Russo',* \\ Stefano Landi iD ${ }^{2, *}$ \\ Stephane Courric iD $^{3}$ \\ 'Department of Management, University \\ of Venice, Venezia, Italy; ${ }^{2}$ Department of \\ Business Administration and \\ Management, University of Verona, \\ Verona, Italy; ${ }^{3}$ Hays Pharma, Paris, France \\ *These authors contributed equally to \\ this work
}

Introduction: Diabetic chronic foot ulcers (DFU) lead to pain, reduced quality of life and represent a severe economic burden for patients and health systems. The clinical results of PRP effectiveness in the treatment of DFU are promising; on the other hand, the costs associated with treating DFUs with PRP are higher than those using standard therapy. Therefore, this study aims to determine the cost-effectiveness of platelet-rich plasma (PRP) therapy compared to standard therapy from the French healthcare system perspective.

Methods: A cost-effectiveness analysis (CEA) was performed using a decision Markov model with a cohort of patients with chronic DFU (duration of $>3$ weeks) with high orthopaedic risk and with ulcers graded 3A according to University of Texas classification. The effectiveness outcomes are reported in terms of quality adjusted life year (QALY). The costs are reported in euro $(€)$ currency evaluated in 2019. A micro-costing approach alongside a clinical study was used to assess resource use. Deterministic sensibility analyses are reported to evaluate the robustness of the results. The analyses were carried out in the French setting.

Results: The incremental cost-effectiveness ratio (ICER) of PRP treatment is -€613/ QALY, which, being lower than zero, indicates the dominance of the PRP therapy. Deterministic and probabilistic sensitivity analysis underlines the main parameter affecting CE results. Lowest number of standard of care weekly medications (from 5 to 3 ) leads to a €622/QALY while increasing PRP weekly medication (from 1 to 4 ) has an ICER of $€ 732 / \mathrm{QALY}$.

Discussion: PRP is a cost-effective or even a cost-saving alternative in the French setting. PRP has higher cost for the complete medication, but, in the absence of wound complications, has the potential to involve lower consumption of resources in the form of routine medication over a 1-year time horizon.

Keywords: platelet-rich plasma, cost-effectiveness analysis, cost-utility analysis, diabetic foot ulcer

\section{Introduction}

Chronic non-healing ulcer is a major health problem and its prevalence in the world ranges from 1.9 to $13.1 \%{ }^{1,2}$ The incidence of chronic ulcers is expected to increase as the population ages and the risk factors for atherosclerotic occlusion such as smoking, obesity and diabetes are increasing. One of the main causes of nonhealing ulcer is diabetes. Diabetic foot ulcer (DFU) is diagnosed in $12-18 \%$ of patients with type 2 diabetes and in $0.6-2 \%$ of patients with type 1 diabetes. ${ }^{3}$ In addition, $70 \%$ of these patients are diagnosed with peripheral neuropathy. ${ }^{4,5}$ Diabetic chronic wounds lead to pain, discomfort, infection, consultations, dressings, hospitalizations, sequelae, sick leave, and poor quality of life. Individuals
Correspondence: Stefano Landi

Department of Business Administration and Management, University of Verona,

Via Cantarane, 24, Verona, 37I29, Italy

Tel +393463287572

Email stefano.landi@univr.it 
with diabetic foot ulcers are susceptible to infection and the healing process is complicated by diabetic neuropathy leading to chronic non-healing ulcers. Consequently, an estimated $12 \%$ of individuals with diabetic foot ulcer require lower extremity amputation. ${ }^{3,8,9}$

Moreover, chronic ulcers also represent a substantial financial burden for the patient and the healthcare system.6,7 The Eurodiale study analysed the direct and indirect annual costs in several European countries (in 14 European centres including Spain, France, the UK, Czech Republic, Germany, Denmark, Sweden and Italy) with a total of 821 patients with DFU. The mean annual cost per patient was $€ 10,091$, hospitalization being the most relevant direct cost. ${ }^{10}$ In the UK, the mean NHS cost of wound care over 12 months was estimated at $£ 7800$ per DFU (of which $13 \%$ was attributable to amputations), ranging from $£ 2140$ to $£ 8800$ per healed and unhealed DFU, respectively, and $£ 16,900$ per amputated wound. ${ }^{11}$ The main element (around 20\%) of total spending is complications related to DFU. ${ }^{12}$ In the US, DFU accounted for $83 \%$ and $96 \%$ of all major and minor amputations related to foot ulcers, respectively, and significantly increased cost of care (DFU: $\$ 1.38$ vs non-DFU: $\$ 0.13$ billion/year; $p$ $<0.001) .{ }^{13}$ Therefore, it is important to evaluate strategies that can ensure better prevention and management of this type of wound to lower the number of amputations.

The goal of ulcer treatment is to obtain wound closure as expeditiously as possible. Conventional treatment for non-healing ulcers includes wound cleansing, necrotic tissue debridement, prevention, diagnosis, and, if necessary, treatment of infection, mechanical off-loading, management of blood glucose levels and local ulcer care with dressing application. ${ }^{3,14}$ Therapeutic procedures to manage DFU are fundamentally based on an adequate covering of the wound, early treatment of the infection, and relief of pressure, with a probability of healing being close to $60 \%$ in 1 year. Despite treatment, many chronic ulcers fail to heal or persist for months/years and/or recur after healing, requiring additional advanced wound care therapies for adequate healing. At 31 months follow up, diabetic foot ulcers have around a $40 \%$ of recurrence and $12.3 \%$ were not healed at the end of the follow-up period. ${ }^{15-17}$ Moreover, at 3 years there is evidence of a $10 \%$ to $20 \%$ rate of amputations. ${ }^{15,18,19,44}$

It has been demonstrated that plasma growth factor (PGF) such as platelet derived growth factor (PDGF) significantly shortens treatment duration and leads to healing in approximately $80 \%$ of wounds. ${ }^{20-24}$ Many authors ${ }^{20-24}$ found that platelet release has improved healing rates if compared with standard remedies. On the other hand, other studies ${ }^{20,21}$ found no major difference in healing outcome of leg ulcers, between treatment groups with platelet release and control groups (placebo). An extensive review was performed by Picard et al 2015. They carried out a PubMed and Cochrane search (1978-2015) including all studies assessing the clinical effect of platelet-rich plasma (PRP) on the healing of diabetic chronic wounds. The screening retrieved 7555 articles and 12 studies were included. Of six randomized studies included, five found significant benefits for the use of PRP on diabetic chronic foot ulcers. The authors concluded that $87.5 \%$ of controlled studies found a significant benefit for the adjunction of PRP to treat chronic diabetic wounds. As PRP may be beneficial, they suggest using it on diabetic ulcers which remain unhealed after standard treatment. ${ }^{25}$ The most recent meta-analyses and review articles have highlighted the therapeutic potential of PRP in chronic wounds with demonstrated benefits in several clinical outcomes, notably complete wound closure, wound surface reduction, scar reduction, and a lowering of incidence of infections. ${ }^{26-34}$

The clinical results of PRP effectiveness in the treatment of DFU are promising considering, however, that the costs associated with treating DFUs with PRP are presumably higher than using standard therapy. Therefore, the implementation of this therapeutic approach in health systems should be based on its cost-effectiveness. Only a few studies have directly dealt with the relationship between the costs and the results of PRP versus standard care and, in addition, no economic evaluations in parallel with clinical studies have been reported. Two studies prior to 2014 found PRP treatment being cost-effective or even dominant compared with usual care. ${ }^{22,35,36}$ Recently, Linertová et al found that, in a 5-year time horizon, PRP treatment for DFU could be considered a cost-effective or even a cost-saving alternative in Spanish healthcare settings, depending on the method of obtaining the PRP (commercial kit versus manual method) and, to a large extent, on the price of the kit used. As a consequence, these authors called for future studies on the effectiveness and costs of specific devices or methods to be used as inputs for more specific cost-effectiveness models. ${ }^{37}$ The objective of this study is to close the gap through a cost-effectiveness analysis, with a 1-year time horizon, of a specific PRP preparation procedure for the treatment of DFU versus standard of care accounting for cost using a micro-costing approach. In order to collect detailed data on resource use, 
material costs and professional time spent on care, our analysis was conducted alongside a clinical study (Le Creusot study, France). ${ }^{38}$ This paper provides more specific evidence and insights for clinical and managerial decision making exploiting a French setting.

\section{Methodology \\ Le Creusot Study}

A randomized controlled trial to evaluate the safety and efficacy of autologous platelet-rich plasma (PRP) gel for stimulating wound healing in chronic deep diabetic foot ulcers (3 A stage according to UT classification) was performed at the Fondation Hôtel-Dieu hospital in Le Creusot, France. $\mathrm{N}=86$ diabetic patients were randomized either to best standard of care (SoC) alone or PRP treatment combined with SoC. Good standard of care was used when appropriate and included debridement, infection control, comorbidities management and off-loading. The two arms were comparable at baseline for age, presence of a neuropathy, antiaggregant treatment, and depth and surface of wounds. ${ }^{38}$

In PRP treatment, gel is obtained from patients' blood by getting a platelet-rich, leukocyte-poor plasma using Regenkit (RegenLab, Switzerland). The commercial kit consists of 3 tubes ( 2 BCT and 1 ATS) that are used to produce a PRP gel.

This is an outpatient clinical protocol: after a 2-week run-in period, if no reduction of wound surface $>20 \%$ was observed, patients were randomized (PRP gel application or control SoC treatment). Patients were treated for 6 weeks maximum (less in case of wound closure). PRP gel arm patients could receive more than one gel application upon investigator decision. Primary efficacy endpoint for wound closure was measured at 6 weeks. Efficacy was also evaluated at follow-up visits at 9 and 12 weeks.

For the PRP arm, only protective compress change was done at home once weekly, while for the control group the dressing change was performed at home once daily.

Forty-six patients were randomized to the PRP gel treatment and 40 to the SoC alone arm. All patients were neuropathic, with deep ulcers mostly located on toe and metatarsal, but also heel and plantar vault. Chronic wound duration was on average more than 6 months.

In terms of results observed at 6 weeks after initiation of treatment, PRP arm shows a complete wound closure in $56.5 \%$ of patients versus $20.0 \%$ in control group, with a statistically significant difference ( $\mathrm{p} \leq 0.001)$. Similarly,
PRP arm reaches $77.3 \%$ wound closure at 12 weeks versus $35.7 \%$ for SoC control group. During the study, data on resource use and costs were collected. In particular, the authors picked for both arms material usage, professional time spent on care and costs for each activity.

\section{Model Description}

The cost-effectiveness analysis was performed using a Markov model, with a hypothetical cohort of patients with chronic DFU (duration of $>3$ weeks) with high orthopaedic risk and with ulcers graded $3 \mathrm{~A}$ (UT classification), meaning deep ulcers down to the bone, including tunnelling and perforating wounds.

Using a decision-analytical model, we defined a structure and within it could insert evidence from this specific clinical study's outcomes (Le Creusot study) as well as from the literature to generalize the results. The advantage of using Markov model is that it is flexible, particularly adapted for modelling chronic diseases spreading over months or years, and deals with ongoing risks and events that might happen more than once over time (recurrence).

The first step was to define DFUs in terms of mutually exclusive states, including all relevant health outcomes, with movement between these states based on transition probabilities. In this study, the Markov model contained six possible health states (2 temporary states, 3 standard states, 1 absorbing state): (1) DFU (first) complete treatment; (2) persistent DFU; (3) completely healed DFU; (4) amputation; (5); post-amputation; and (6) death from any cause and because of surgery (see Figure 1). At the beginning of the model, patients with DFUs would receive the first treatment according to one of the following strategies: PRP combined with best SoC or Soc alone. Hypothetical patients started in state 1 and moved into predefined health states. Each clinical state had an associated cost and effectiveness estimate. Hypothetical patients accumulated costs and QALYs associated with the time spent in each clinical state during the simulated 3-month period. The cycle length was 1 week and the time horizon was 1 year.

In the first state, patients receive their first complete treatment. The first state is a temporary state, therefore patients receive value of cost and utilities for the first treatment and then go with a $100 \%$ probability to the second state. In state 2 (persistent DFU), they receive the weekly care. For PRP treatment, there is a certain probability of needing a new complete treatment that is more costly than the weekly care. We modelled the necessity of some patients having more than one complete treatment 


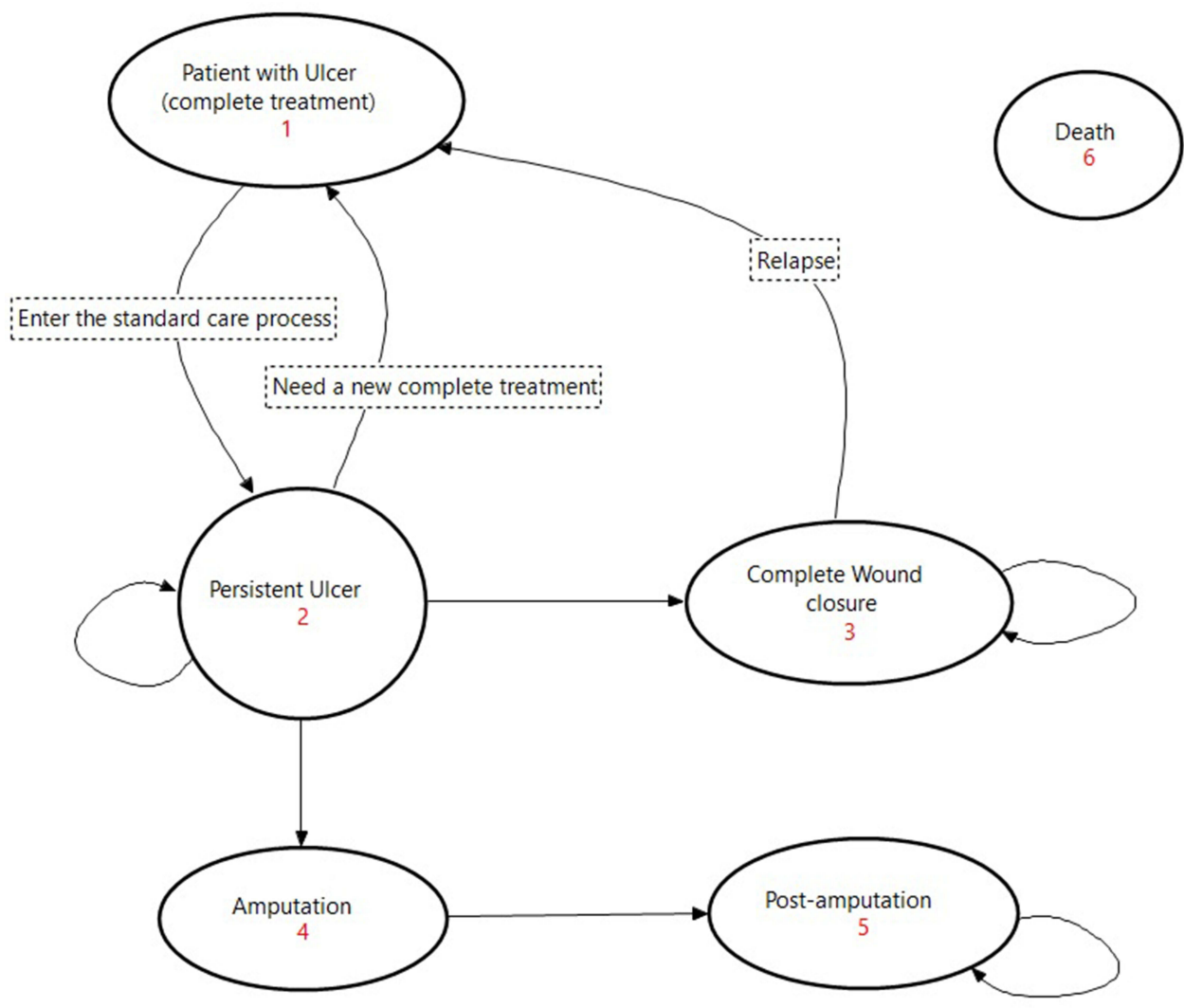

Figure I Markov model of diabetic foot ulcer. States are identical for usual and PRP care.

using a transient event rather than a state. Therefore, we used transition rewards so that a one-time cost was associated with a patient having a second treatment. Although the need of a new treatment is not a state itself, and it has no effect on state transition, it may have costs (and/or disutilities) associated with it. For traditional care, this possibility is not allowed since the dressing change is performed daily until the wound is healed.

In state 2, for PRP arm, the home nurse opens the dressing and checks the cleanliness of the primary dressing (according to Le Creusot study protocol, this is performed once a week in the absence of complications). If the dressing is clean, the nurse will operate the simple replacement of the secondary dressing. Otherwise, a second complete treatment is planned. State 2, for usual treatment arm, draws the complex dressing (dressing change) performed by a home nurse every day who carries out the cleaning and dressing procedure 5 days per week.

In state 3, patients receive costs and utility of a complete healing of their ulcer. As scientific evidence shows, there is some probability that diabetic patients can have a new occurrence, thus they can move from the state "complete wound disclosure", through relapse, to the necessity of new medications. State 6 is the absorbing state and collects individuals dying from any cause (life table) and because of surgery. Results are reported in terms of incremental cost-effectiveness ratio (ICER).

\section{Cost Inputs}

All costs associated with health states and transition costs in the Markov model were measured in euros for 2019, as presented in Table 1. Costs were estimated through a micro-costing approach alongside the resources used in Le Creusot study. These costs are from the perspective of the French healthcare system.

For the cost of dressings, we have made a few estimations to get the average price of a simple dressing (PRP group) and a complex dressing ( $\mathrm{SoC}$ group). Simple dressing made use of Jelonet, Adaptic, Grassolind plus secondary dressing as serum. Complex dressing, depending on wound characteristics, made use of different families of products. From Le Creusot study we have a rate of utilization of $70 \%$ of hydrocellular dressing (Mepilex, Ialuset, Urgotull), $20 \%$ on superabsorbant hydrocellular dressing (Cutimed, Wilsabord, Sorbact) and 10\% using Urgostart. In Appendix Table 1, we summarize the main parameters 
Table I Data Used in the Model: Costs

\begin{tabular}{|c|c|c|c|c|}
\hline \multirow[t]{2}{*}{ Parameters } & \multirow{2}{*}{$\begin{array}{l}\text { Base Case } \\
\text { Value }(€)\end{array}$} & \multicolumn{2}{|c|}{ Range } & \multirow[t]{2}{*}{ Data Source } \\
\hline & & Min & Max & \\
\hline Nurse/minute $(€)$ & 0.30 & 0.26 & 0.33 & $\begin{array}{l}\text { Collective agreement } 2019 \\
\text { Min: (junior nurse) } \\
\text { Max: (senior nurse) }\end{array}$ \\
\hline Blood sampling tariff (AMI I,5) & 4.725 & & & $\begin{array}{l}\text { Conventional tariff applicable to the activity of } \\
\text { nurses (Ameli.fr) }\end{array}$ \\
\hline Time resources & & & & \\
\hline $\begin{array}{l}\text { Minutes to prepare PRP and dressing (complete } \\
\text { procedure)/day }\end{array}$ & 30 minutes & 25 & 35 & Le Creusot study ${ }^{38}$ \\
\hline $\begin{array}{l}\text { Minutes for checking the wound treated with PRP/ } \\
\text { weekly }\end{array}$ & 15 minutes & 10 & 20 & Le Creusot study ${ }^{38}$ \\
\hline Minutes to prepare Hydrocolloid and dressing/daily & 20 minutes & 15 & 25 & Le Creusot study ${ }^{38}$ \\
\hline Material used & & & & \\
\hline RegenKit BCT-I (X 2 units) & 55 & 44 & 75 & Market price \\
\hline RegenKit ATS (X I unit) & 55 & 44 & 75 & Market price \\
\hline Cleaning and secondary dressing* & & & & \\
\hline PRP treatment & 7.33 & 5.87 & 8.79 & $\begin{array}{l}\text { Use PRP I day/week } \\
\text { Le Creusot study }{ }^{38}\end{array}$ \\
\hline Standard of care & 12.9 & 10.3 & 15.5 & $\begin{array}{l}\text { Use SoC } 5 \text { days/week } \\
\text { Le Creusot study }{ }^{38}\end{array}$ \\
\hline Amputation & & & & \\
\hline Surgery cost & 9,721 & 5,319 & $14^{\prime} 000$ & Technical hospital information agency ${ }^{\S}$ \\
\hline Annual post-surgery costs & 800 & & & Tchero et al ${ }^{42}$ \\
\hline PRP one complete procedure total cost & 186 & & & Le Creusot study ${ }^{38}$ \\
\hline PRP weekly routine check total cost & 7.33 & 5.87 & 8.79 & Le Creusot study ${ }^{38}$ \\
\hline Hydrocolloid procedure weekly total cost & 64.5 & 51.5 & 77.5 & Le Creusot study ${ }^{38}$ \\
\hline
\end{tabular}

Note: *The total cost for one-day routine medication is reported (including nurse time and materials).

Abbreviation: ${ }^{\S} \mathrm{ATIH}$, Agence technique de l'information hospitalière (https://www.atih.sante.fr/).

used. The details of the calculations on the materials used are included in the Excel file "Couts Materiels Hopital".

In state 1, PRP cost is due to: the use of regenkit BCT1 (X2) and ATS (X1) + cleaning procedure + the medication + nurse time. The cost of comparator is due to the cost of complex dressing + cleaning procedure + nurse time (state 1: DFU complete treatment: PRP arm: €186; comparator: €12.6). In state 2, PRP cost of $€ 7.3$ is due to the repair of the medication + nurse time. The cost of comparator is due to the cost of complex dressing + cleaning procedure + nurse time multiplied 5 times per week (state 2: persistent DFU (PRP: €7.3; comparator: €63).

\section{Utility Inputs}

Quality of life assessments for six possible health states (see model description) were based on general health status profiles, which are less sensitive than illness-specific scales but allowed us to analyse and compare results outside the context of a certain disease. A variety of generic preference-based measures have been developed; the most used questionnaires include the EuroQol EQ-5D, the Short Form 6D (SF-6D) and the Health Utilities Index (HUI). Once completed, the questionnaires generate a score using an algorithm based on values that have been obtained from a sample of the general public. ${ }^{40}$ The values are the healthrelated quality of life (HRQoL) and measure the utility from living in a specific health state. Health states assume HRQoL values between 0 (dead) and 1 (perfect health); negative values are possible when the health status is considered worse than death. QALYs are assessed by combining the weights calculated for health states alongside the time spent in those health states. QALYs represent the number of years lived in perfect health. The advantage of their use is the possibility to compare results among 
Table 2 Data Used in the Model: Effectiveness (at 3 Months)

\begin{tabular}{|c|c|c|c|c|}
\hline \multirow[t]{2}{*}{ Health State } & \multirow[t]{2}{*}{ Base Case (QALYs) } & \multicolumn{2}{|c|}{ Range } & \multirow[t]{2}{*}{ References } \\
\hline & & Min & $\operatorname{Max}$ & \\
\hline No active ulcer & 0.84 & 0.81 & 0.87 & Redekop et al $2004^{41}$ \\
\hline Active ulcer & 0.70 & 0.66 & 0.75 & Redekop et al $2004^{41}$ \\
\hline One foot amputated & 0.68 & 0.63 & 0.72 & Redekop et al $2004^{41}$ \\
\hline Amputation & 0.31 & & & Barshes et al $201 \mathrm{I}^{43}$ \\
\hline
\end{tabular}

pathologies and among willingness to pay thresholds for health outcomes.

In this analysis, quality of life assessments for the six possible health states were based on the generic EuroQol instrument and obtained from a previous published study, as presented in Table $2{ }^{41}$ Redekop et al used a time tradeoff method to estimate the utility weights associated with a range of health states related to DFUs and their complications. This source was also used previously to provide the quality-of-life estimates for a cost-effectiveness analysis of a negative pressure device for the treatment of DFUs. QALYs were calculated by multiplying the quality-of-life utility weight for one health state by the number of years staying in that state.

\section{Probabilities}

Probabilities of healing were informed by the proportion of ulcers completely healed at the 12-week time point (Le Creusot study data), $77.3 \%$ for PRP care and $35.1 \%$ for usual care. Whereas Le Creusot study showed strong results in favor of PRP, in our cost-effectiveness analysis we decided to use a conservative approach. The model parameters were from a recent meta-analysis based on five existing clinical trials; the healing success was $58.33 \%$ for PRP and $50.31 \%$ for usual care ${ }^{28}$ (see Table 3).
In Le Creusot study, a total of 27 patients had one injection, 17 had two injections and 2 patients had 3 PRP applications. On average, patients received 1.46 PRP treatments. Therefore, we modelled a conservative probability of 0.5 per cycle to receive a second complete treatment (from Le Creusot study, the probability of having a second complete treatment is 0.2 ).

To generalize the model, the risk of amputation and relapse were included. Amputation is a temporary state leading to the post-amputation state. The probability of amputation was taken from the Moulik study ${ }^{18}$ and adjusted according to the model cycles ( 1 week). The new occurrence probability was taken from the Eurodiale study. $^{44}$

All annual and three-month probabilities were transformed to weekly probabilities by the formula proposed by Briggs: ${ }^{39}$

$$
\operatorname{tp}_{1}=1-\left(1-\mathrm{tp}_{\mathrm{t}}\right)^{1 / \mathrm{t}}
$$

where $\mathrm{tp}_{1}$ is the weekly transition probability we wish to estimate and $\mathrm{tp}_{\mathrm{t}}$ is the overall probability over time, $\mathrm{t}$.

\section{Sensitivity Analysis}

Deterministic sensitivity analysis has been conducted to assess the impact of the uncertainty of the parameters used in the model on the results. Deterministic sensitivity

Table 3 Data Used in the Model: Probability of Healing (at 3 Months)

\begin{tabular}{|l|l|l|l|l|}
\hline \multirow{2}{*}{ Parameters } & \multirow{2}{*}{ Base Case (\%) } & \multicolumn{2}{|c|}{ Range } & \multirow{2}{*}{ Max } \\
\cline { 3 - 5 } & & \multicolumn{1}{|c|}{ Min } & & \multirow{2}{*}{ References } \\
\hline $\begin{array}{l}\text { Probability of healing } \\
\text { PRP }\end{array}$ & 0.58 & & 0.69 & Del Pino-Sedeño $2019^{28}$ \\
Hydrocolloid & 0.50 & 0.46 & 0.60 & Eurodiale, Dubský et al 2013 \\
\hline Recurrence & 0.068 & 0.40 & 0.081 & Moulik study $^{18}$ \\
\hline Amputation & 0.014 & 0.011 & 0.017 & Moulik study $^{18}$ \\
\hline Surgery mortality & 0.0029 & - & - & \\
\hline
\end{tabular}


analyses are useful to deal with different uncertainties, whereby: (i) model uncertainty means every model is a simplification of the reality, (ii) parameter uncertainty is an estimation of costs and effectiveness, and (iii) heterogeneity is the individual variability between patients. Therefore, the sensitivity analyses have been run for the most important parameters of the model (Tables 1-3). Deterministic sensitivity analysis (DSA) has been run for every min-max scenario of any parameters. In detail, oneway DSA and tornado analysis have been run for every parameter where a min and max scenario is reported.

Probabilistic sensitivity analysis (PSA) was performed through a Monte Carlo simulation, performing 10,000 cases, to assess the uncertainty around the ICER and the probability of the PRP therapy to be cost-effective at different willingness to pay thresholds. A probability distribution was assigned at each model input parameter to describe the different values the parameter can have with different probabilities. The effectiveness and probabilities have been modelled with beta distributions; costs were represented as Gamma distributions as recommended in literature. ${ }^{45}$ For the parameters cited in literature where it was not estimated standard error, it was assumed a general standard error of $25 \%$ of the mean value. ${ }^{39}$

All analyses were perfomed using TreeAge Pro 2021.

\section{Results}

In the base case scenario PRP treatment results in cost savings. The average cost per QALY is around $€ 188$ for comparator and $€ 181$ for PRP, respectively. The ICER of PRP treatment is $-€ 613 / \mathrm{QALY}$, which, being lower than zero, indicates the dominance of the PRP therapy.

In order to know how each parameter influences the results, a one-way sensitivity analysis was performed for the main parameters. The tornado analysis shows the collection of one-way sensitivity analyses for the main parameters (see Figure 2). The DSA results are consistent with the base case scenario. However, for some parameters the PRP treatment is not cost-saving but having a positive ICER can be cost-effective according to the willingness to pay thresholds used.

The most sensitive parameters are the PRP arm probability of needing a new complete treatment and the number of weekly medications carried out for both. The analysis shows that the main difference in costs is due to the daily nurse visits and dressing changes for the comparator therapy, while for PRP therapy we have only one visit per week. The PRP therapy cost is higher in the first

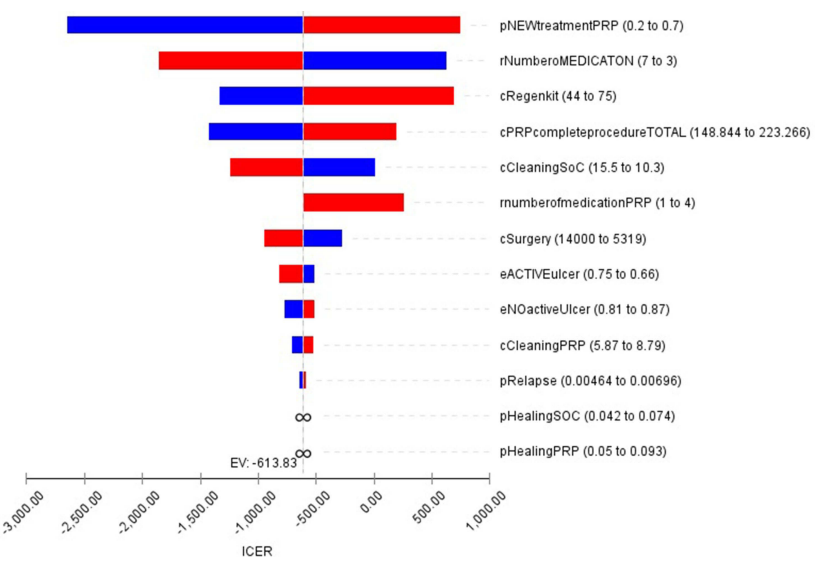

Figure 2 Tornado analysis.

week $(€ 186)$ due to the expense for medical devices and nurse time, but it decreases at few euros over the following weeks because only $50 \%$ of patients per week need a new complete medication. Therefore, the PRP branch needs less nurse time and a reduced use of materials resulting in a cost saving in respect of the standard of care $(\mathrm{SoC})$ alone. Initially, PRP treatment is more costly but at each cycle the cumulative costs become lower than for usual care and from week 7 the comparator become the most expensive. This trend is due mainly to less frequency of medications for PRP and to a better healing probability (Figure 3).

Changing the PRP arm's probability of needing a new complete treatment from 0.5 to 0.7 leads PRP strategy to be no longer cost-saving, with an ICER of €732/QALY. Including a lowest number of $\mathrm{SoC}$ weekly medications (from 5 to 3) leads to a €622/QALY, while increasing PRP weekly medication (from 1 to 4 ) has an ICER of $€ 732$ QQALY. Considering common thresholds of around $€ 20 / 30,000$, PRP is a cost-effective option.

Other sensitive parameters that lead PRP therapy to be

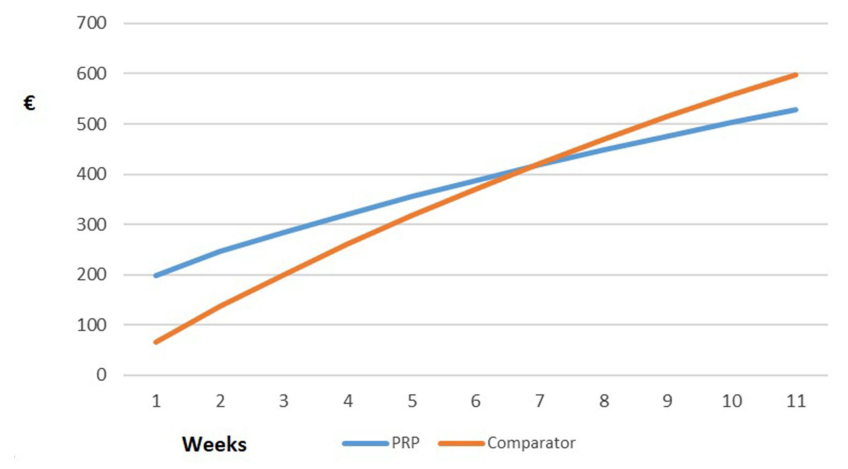

Figure 3 Cumulative cost per week of the two therapies. 


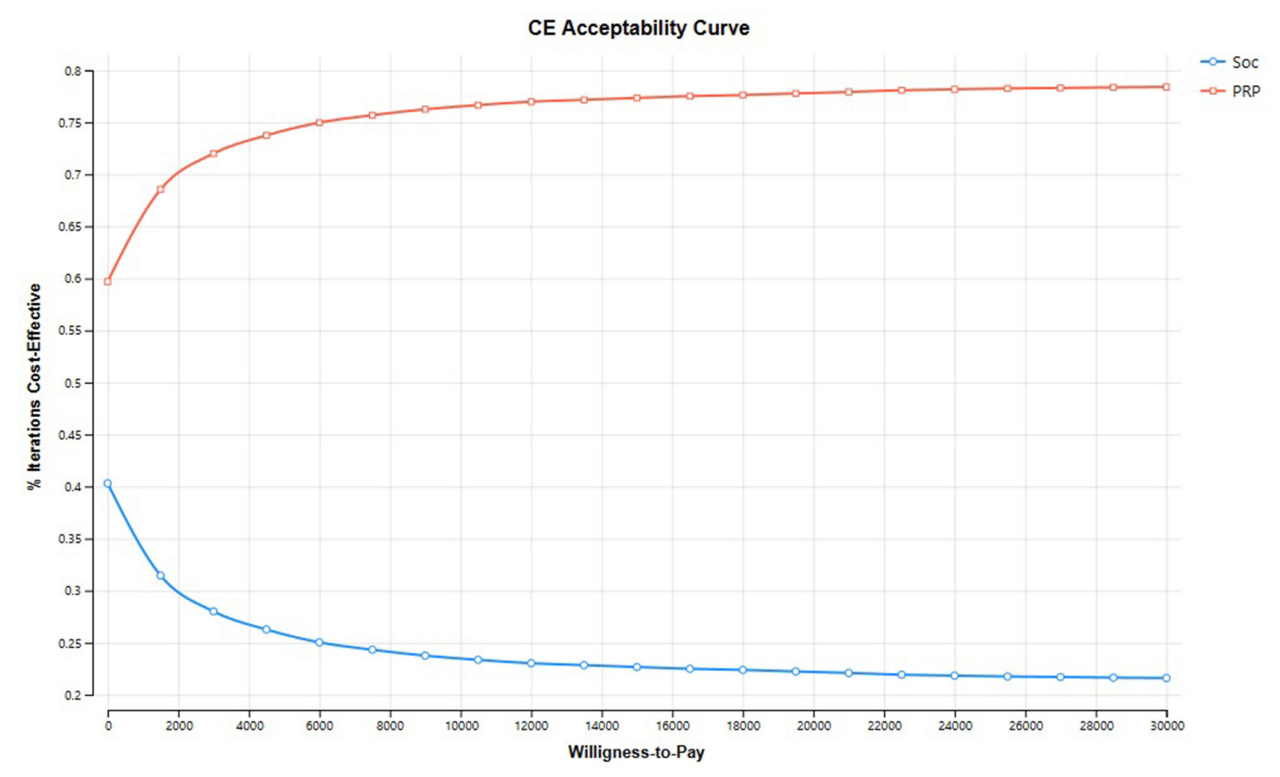

Figure 4 Cost-effectiveness acceptability curve of PRP vs SoC under various WTP thresholds.

cost-effective and not cost-saving are the cost of the kit used and the total cost of the complete procedure.

The probabilistic sensitivity analysis was performed through a Monte Carlo simulation considering 10,000 scenarios (or cases). All the parameters and variables of the model vary according to the assigned distribution. Figure 4 shows the corresponding acceptability curve with the WTP thresholds. For every WTP threshold, the percentage of cases in favor of PRP or HA is shown, where the percentage of cost-effective iterations is derived from a probabilistic sensitivity analysis performed through the Monte Carlo simulation. For example, at $€ 10,000$ / QALY there is a $77 \%$ probability for PRP treatment to be cost-effective.

\section{Conclusions}

The present study evaluates the cost-effectiveness of PRP treatment compared with usual management of DFU patients in patients with chronic DFU (duration of $>3$ weeks) with high orthopaedic risk and ulcers graded $3 \mathrm{~A}$ (UT classification). This work was conducted along with a clinical study (Le Creusot study) allowing a micro-costing approach in the assessment of resource utilization. Conservative data on effectiveness obtained from a recent meta-analysis were considered (the healing success was $58.33 \%$ for PRP and $50.31 \%$ for usual care). ${ }^{28}$ Under these assumptions, the PRP therapy resulted in cost-savings in the base case scenario.
The number of weekly medications for both treatments and the PRP arm probability of needing a new complete treatment influence the results because the PRP, after the first complete medication, needs only one intervention per week in comparison to five for usual care. PRP has higher cost for the complete medication but, in the absence of complications (nurse checks once weekly), only the change of secondary dressing is needed. In the absence of healing signs, the physician can schedule a second PRP application. The probability used in the model for a second PRP complete treatment is $50 \%$ weekly. Therefore, PRP has the potential to involve lower consumption of resources (both nurse time and materials). As shown in sensitivity analysis, considering three medications per week (instead of five) for usual care, PRP is no longer the cost-saving therapy but with a $€ 622 / \mathrm{QALY}$ is costeffective (ICER inferior to the threshold of $€ 30,000$ / QALY). Our results confirm the findings of identified economic evaluations ${ }^{35-37}$ which found the PRP treatment being cost-effective or even dominant compared with usual care. The three works before 2014 have limited methodological quality and do not provide sufficient details of the evaluated procedures. A recent study from a Spanish setting found that, in a 5-year time horizon, PRP treatment for DFU could be considered a cost-effective or even a cost-saving alternative. However, there are few economic evaluations probably because of the scarcity of robust clinical trials and for the wide heterogeneity of the population enrolled in the studies. 
This work is not free from limitations. It used cost data specific to the French setting because it is rooted in Le Creusot study. It helps to give more details regarding the PRP vs SoC procedure and allows a micro-costing approach, but it can be less generalizable to other contexts. For example, even if the tornado analysis showed consistent results, the number of PRP complete treatments may influence the outcome of the model and thus calls for further analysis. Moreover, the results need to be interpreted in light of a limited level of evidence complicated by heterogeneity in the characteristics of the patients treated and adding to these factors also the procedures used for $\mathrm{PRP} /$ usual care therapies such as, for example, the number of medications and the type of materials used. Therefore, more high-quality randomized controlled trials, possibly with longer term follow up, are required.

In conclusion, considering limitations, the PRP treatment for DFU could be considered a cost-effective or even a cost-saving alternative in the French setting.

\section{Ethical Statement}

This study does not contain human/animal test subjects.

\section{Acknowledgments}

The paper is the advancement of a previous working paper published on the website of Department of Management, University Cà Foscari Venice: Salvatore Russo \& Stefano Landi, 2020. "Cost-effectiveness analysis for the treatment of diabetic foot ulcer in France: PRP vs standard of care," Working Paper n.4/2020, August 2020, ISSN:2239-2734. This research was supported by University Ca'à Foscari Venezia through a grant provided by Regenlab SA to SL. Salvatore Russo and Stefano Landi are co-first authors for this study.

\section{Author Contributions}

All authors made a significant contribution to the work reported. In detail, all authors worked on the conception, study design, and interpretation. SC worked on acquisition of data, SL and SR on execution and analysis. All authors took part in drafting, revising or critically reviewing the article; gave final approval of the version to be published; have agreed on the journal to which the article has been submitted; and agree to be accountable for all aspects of the work.

\section{Disclosure}

SC formerly worked for Regen Lab as external consultant. The authors report no other conflicts of interest in this work.

\section{References}

1. Rayner R, Carville K, Keaton J, et al. Leg ulcers: atypical presentations and associated co-morbidities. Wound Pract Res. 2009;17 (4):168-185

2. Agale SV. Chronic leg ulcers: epidemiology, aetiopathogenesis, and management. Ulcers. 2013;Article ID 413604:9.

3. Greer N, Foman N, Dorrian J, et al. Advanced wound care therapies for nonhealing diabetic, venous, and arterial ulcers: a systematic review. VAESP Project \#09-009; 2012.

4. Abbott CA, Carrington AL, Ashe H, et al. The North-West Diabetes Foot Care Study: incidence of, and risk factors for, new diabetic foot ulceration in a community - based patient cohort. Diabet Med. 2002;19:377-384. doi:10.1046/j.1464-5491.2002.00698.x

5. Abbott CA, Vileikyte L, Williamson S, et al. Multicenter study of the incidence of and predictive risk factors for diabetic neuropathic foot ulceration. Diabetes Care. 1998;21:1071. doi:10.2337/diacare.2 1.7.1071

6. Siersma V, Thorsen H, Holstein PE, et al. Importance of factors determining the low health-related quality of life in people presenting with a diabetic foot ulcer: the Eurodiale study. Diabet Med. 2013;30:1382. doi:10.1111/dme.12254

7. Hopkins RB, Burke N, Harlock J, et al. Economic burden of illness associated with diabetic foot ulcers in Canada. BMC Health Serv Res. 2015;15:13. doi:10.1186/s12913-015-0687-5

8. Buckley CM, O'Farrell A, Canavan RJ, et al. Trends in the incidence of lower extremity amputations in people with and without diabetes over a five-year period in the Republic of Ireland. PLoS One. 2012;7: e41492. doi:10.1371/journal.pone.0041492

9. Brem H, Tomic-Canic M. Cellular and molecular basis of wound healing in diabetes. J Clin Invest. 2007;117:1219-1222. doi:10.1172/ JCI32169

10. Prompers L, Huijberts M, Schaper N, et al. Resource utilisation and costs associated with the treatment of diabetic foot ulcers. Prospective data from the Eurodiale Study. Diabetologia. 2008;51:1826-1834. doi:10.1007/s00125-008-1089-6

11. Guest JF, Fuller GW, Vowden P. Diabetic foot ulcer management in clinical practice in the UK: costs and outcomes. Int Wound J. 2018;15 (1):43-52. doi:10.1111/iwj.12816

12. Singh N, Armstrong DG, Lipsky BA. Preventing foot ulcers in patients with diabetes. JAMA. 2005;293:217-228. doi:10.1001/ jama.293.2.217

13. Hicks CW, Selvarajah S, Mathioudakis N, et al. Burden of infected diabetic foot ulcers on hospital admissions and costs. Ann Vasc Surg. 2016;33:149-158. doi:10.1016/j.avsg.2015.11.025

14. Anderson I. Aetiology, assessment and management of leg ulcers. Wound Essent. 2006;1:20-36.

15. Winkley K, Stahl D, Chalder T, Edmonds ME, Ismail K. Risk factors associated with adverse outcomes in a population based prospective cohort study of people with their first diabetic foot ulcer. J Diabetes Complications. 2007;21:341-349. doi:10.1016/j.jdiacomp.20 07.09 .004

16. Pound N, Chipchase S, Treece K, Game F, Jeffcoate W. Ulcer-free survival following management of foot ulcers in diabetes. Diabet Med. 2005;22:1306-1309. doi:10.1111/j.1464-5491.2005.01640.x

17. Peters EJG, Armstrong DG, Lavery LA. Risk factors for recurrent diabetic foot ulcers: site matters. Diabetes Care. 2007;30:2077-2079. doi: $10.2337 / \mathrm{dc} 07-0445$ 
18. Moulik PK, Mtonga R, Gill GV. Amputation and mortality in newonset diabetic foot ulcers stratified by etiology. Diabetes Care. 2003;26(2):491-494. doi:10.2337/diacare.26.2.491

19. Martinez-Zapata MJ, Martí-Carvajal AJ, Solà I, et al. Autologous platelet rich plasma for treating chronic wounds. Cochrane Database Syst Rev. 2012;10:Art. No.: CD006899. doi:10.1002/14651858. CD006899.pub2

20. Saad Setta H, Elshahat A, Elsherbiny K, Massoud K, Safe I. Plateletrich plasma versus platelet-poor plasma in the management of chronic diabetic foot ulcers: a comparative study. Int Wound J. 2011;8(3):307-312. doi:10.1111/j.1742-481X.2011.00797.x

21. O’Meara SM, Cullum NA, Majid M, Sheldon TA. Systematic review of antimicrobial agents used for chronic wounds. $\mathrm{Br} J$ Surg. 2001;88:4-21. doi:10.1046/j.1365-2168.2001.01631.x

22. Kantor J, Margolis DJ. Treatment options for diabetic neuropathic foot ulcers: a cost-effectiveness analysis. Dermatol Surg. 2001;27:347-351. doi:10.1046/j.1524-4725.2001.00280.x

23. Suthar M, Gupta S, Bukhari S, Ponemone V. Treatment of chronic non-healing ulcers using autologous platelet rich plasma: a case series. J Biomed Sci. 2017;24:16. doi:10.1186/s12929-017-0324-1

24. Senet P, Bon FX, Benbunan M, et al. Randomized trial and local biological effect of autologous platelets used as adjuvant therapy for chronic venous leg ulcers. J Vasc Surg. 2003;38:1342-1348. doi:10.1016/S0741-5214(03)00908-X

25. Picard F, Hersant B, Bosc R, Meningaud JP. The growing evidence for the use of platelet-rich plasma on diabetic chronic wounds: a review and a proposal for a new standard care. Wound Repair Regen. 2015;23(5):638-643. doi:10.1111/wrr.12317

26. Conde-Montero E, de la Cueva Dobao P, Martínez González JM. Platelet-rich plasma for the treatment of chronic wounds: evidence to date. Chronic Wound Care Manag Res. 2017;4:107-120. doi:10.2147/CWCMR.S118655

27. Hirase T, Ruff E, Surani S, Ratnani I. Topical application of plateletrich plasma for diabetic foot ulcers: a systematic review. World $J$ Diabetes. 2018;9(10):172-179. doi:10.4239/wjd.v9.i10.172

28. Del Pino-Sedeño T, Trujillo-Martín MM, Andia I, et al. Platelet-rich plasma for the treatment of diabetic foot ulcers: a meta-analysis. Wound Repair Regen. 2019;27(2):170-182. doi:10.1111/wrr.12690

29. Hsieh TS, Chiu WK, Yang TF, Wang HJ, Chen C. A meta-analysis of the evidence for assisted therapy with platelet-rich plasma for atrophic acne scars. Aesthetic Plast Surg. 2019;43(6):1615-1623. doi:10.1007/s00266-019-01471-w

30. Shen Z, Zheng S, Chen G, et al. Efficacy and safety of platelet-rich plasma in treating cutaneous ulceration: a meta-analysis of randomized controlled trials. J Cosmet Dermatol. 2019;18(2):495-507. doi:10.1111/jocd.12853

31. Ding H, Fu XL, Miao WW, Mao XC, Zhan MQ, Chen HL. Efficacy of autologous platelet-rich gel for diabetic foot wound healing: a meta-analysis of 15 randomized controlled trials. Adv Wound Care (New Rochelle). 2019;8(5):195-207. doi:10.1089/wound.2018.0861

32. Li Y, Gao Y, Gao Y, et al. Autologous platelet-rich gel treatment for diabetic chronic cutaneous ulcers: a meta-analysis of randomized controlled trials. J Diabetes. 2019;11(5):359-369. doi:10.1111/17530407.12850

ClinicoEconomics and Outcomes Research

\section{Publish your work in this journal}

ClinicoEconomics and Outcomes Research is an international, peerreviewed open-access journal focusing on Health Technology Assessment, Pharmacoeconomics and Outcomes Research in the areas of diagnosis, medical devices, and clinical, surgical and pharmacological intervention. The economic impact of health policy and health systems

Submit your manuscript here: https://www.dovepress.com/clinicoeconomics-and-outcomes-research-journa
33. Xia Y, Zhao J, Xie J, Lv Y, Cao DS. The efficacy of platelet-rich plasma dressing for chronic nonhealing ulcers: a meta-analysis of 15 randomized controlled trials. Plast Reconstr Surg. 2019;144(6):14631474. doi:10.1097/PRS.0000000000006281

34. Hu Z, Qu S, Zhang J, et al. Efficacy and safety of platelet-rich plasma for patients with diabetic ulcers: a systematic review and meta-analysis. Adv Wound Care (New Rochelle). 2019;8(7):298-308. doi:10.1089/wound.2018.0842

35. Dougherty EJ. An evidence-based model comparing the cost-effectiveness of platelet-rich plasma gel to alternative therapies for patients with nonhealing diabetic foot ulcers. Adv Skin Wound Care. 2008;21(12):568-575. doi:10.1097/01.ASW.0000323589.27605.71

36. Cobos Campos R, Parraza Diez N, Aizpuru Barandiaran F. Plateletrich plasma in skin ulcer treatment. Wounds. 2013;25(9):256-262.

37. Linertová R, Del Pino-Sedeño T, Pérez LG, et al. Cost-effectiveness of platelet-rich plasma for diabetic foot ulcer in Spain [published online ahead of print, $2020 \mathrm{Feb} 10]$. Int $J$ Low Extrem Wounds. 2020;1534734620903239. doi:10.1177/1534734620903239.

38. Clavel S, Albache N, Labrut H, Robu E, Denizot C. Autologous platelet gel: an help in chronic deep diabetic foot ulcers treatment in "2019 Diabetic Foot Conference Abstracts". J Diabetes Sci Technol. 2020;14(3):601-678. doi:10.1177/1932296819897643

39. Briggs A, Claxton K, Sculpher M. Decision Modelling for Health Economic Evaluation. Oxford: Oxford University Press; 2006.

40. van Hout B, Janssen MF, Feng YS, et al. Interim scoring for the EQ5D-5L: mapping the EQ-5D-5L to EQ-5D-3L value sets. Value Health. 2012;15(5):708-715. doi:10.1016/j.jval.2012.02.008

41. Redekop WK, Stolk EA, Kok E, Lovas K, Kalo Z, Busschbach JJV. Diabetic foot ulcers and amputations: estimates of health utility for use in cost-effectiveness analyses of new treatments. Diabetes Metab. 2004:30:549-556. doi:10.1016/S1262-3636(07)70154-4

42. Tchero H, Kangambega P, Lin L, et al. Cost of diabetic foot in France, Spain, Italy, Germany and United Kingdom: a systematic review. Ann Endocrinol (Paris). 2018;79(2):67-74. doi:10.1016/j. ando.2017.11.005

43. Barshes NR, Belkin M; MOVIE Study Collaborators. A framework for the evaluation of "value" and cost-effectiveness in the management of critical limb ischemia. J Am Coll Surg. 2011;213(4):552-66. e5. doi:10.1016/j.jamcollsurg.2011.07.011

44. Dubský M, Jirkovská A, Bem R, et al. Risk factors for recurrence of diabetic foot ulcers: prospective follow-up analysis in the Eurodiale subgroup. Int Wound J. 2013;10(5):555-561. doi:10.1111/j.1742481X.2012.01022.X

45. Briggs AH, Goeree R, Blackhouse G, O’Brien BJ. Probabilistic analysis of cost-effectiveness models: choosing between treatment strategies for gastroesophageal reflux disease. Med Decis Making. 2002;22(4):290-308. doi:10.1177/027298902400448867 organization also constitute important areas of coverage. The manuscript management system is completely online and includes a very quick and fair peer-review system, which is all easy to use. Visit http://www.dovepress.com/testimonials.php to read real quotes from published authors. 